\title{
Real-time detection of highly oxidized organosulfates and BSOA marker compounds during the F-BEACh 2014 field study
}

Martin Brüggemann et al.

Correspondence to: Thorsten Hoffmann (t.hoffmann@uni-mainz.de)

The copyright of individual parts of the supplement might differ from the CC-BY 3.0 licence. 


\section{S-1. UHPLC-(-)ESI-HRMS analysis of filter samples}

\section{S-1.1 Experimental details for the filter analysis}

Filter samples were taken twice a day by passing an air flow of $27.5 \mathrm{~L} \mathrm{~min}^{-1}$ from $\sim 6 \mathrm{~m}$ above ground $\left(\mathrm{PM}_{2.5}\right)$ through tetrafluorethylene-coated borosilicate filters $(70 \mathrm{~mm}$, Pallflex T60A20, Pall Life Science, USA). The sampling time was $\sim 8$ hours for daytime filters ( 9 a.m. -5 p.m.) and $\sim 16$ hours for nighttime filters ( 5 p.m. -9 a.m.). After sampling, the filters were stored at $<-18{ }^{\circ} \mathrm{C}$ until analysis. Blank filters were taken every 2 to 3 days by placing a filter into the filter holder for $\sim 20$ min without sample flow.

For the extraction procedure one half of a filter sample was cut into pieces and $1.5 \mathrm{~mL}$ of a methanol/water solution (9:1) were added as extracting agent. Then, the sample was sonicated for $30 \mathrm{~min}$. The extract was transferred into a separate glass vial and the filter sample extracted three more times in the same way. The combined extracts were then evaporated to dryness under a gentle stream of nitrogen at $50{ }^{\circ} \mathrm{C}$. Afterwards, the residue was dissolved in $200 \mu \mathrm{L}$ using a solution of acetonitrile/water (2:8). To compensate for losses during the sample processing, i.e. extraction efficiency and evaporation, an average recovery rate was determined for pinic acid, which served as a surrogate for the quantification of other monoterpene oxidation products. Here, an average recovery rate of $85 \%$ was found and applied to the detected organic compounds. It should also be noted that differences in extraction efficiencies and matrix effects might have had a significant effect on the observed signal abundances, which is an inherent problem for aerosol analysis by LC-MS, as recently discussed by Riva et al. (2016) for measurements of organosulfates from gas-phase oxidation of alkanes.

For the LC separation of the filter extracts a UHPLC system (Dionex UltiMate 3000, Thermo Scientific, Germany) equipped with a Hypersil Gold column $(\mathrm{C} 18,50 \times 2.0 \mathrm{~mm}, 1.9 \mu \mathrm{m}$, Thermo Scientific, Germany) was used. The injection volume was $20 \mu \mathrm{L}$ per run and each sample was measured in triplicate. As eluents served a solution of ultrapure water with $2 \%$ acetonitrile and $0.04 \%$ formic acid (eluent A) and a solution of acetonitrile with $2 \%$ of ultrapure water (eluent B). At a flow rate of $500 \mu \mathrm{L} \mathrm{min}{ }^{-1}$ the following gradient was used to optimize the separation: $5 \% \mathrm{~B}$ at $0.00 \mathrm{~min}, 5 \% \mathrm{~B}$ at $0.50 \mathrm{~min}, 20 \% \mathrm{~B}$ at $1.00 \mathrm{~min}, 20 \% \mathrm{~B}$ at $1.50 \mathrm{~min}, 90 \% \mathrm{~B}$ at $2.00 \mathrm{~min}, 90 \% \mathrm{~B}$ at $4.00 \mathrm{~min}, 5 \% \mathrm{~B}$ at $4.05 \mathrm{~min}$, and $5 \% \mathrm{~B}$ at $4.10 \mathrm{~min}$. The UHPLC system was coupled to a Q-Exactive mass spectrometer (Thermo Scientific, Germany) which was used to obtain high resolution mass spectra (resolving power of $\mathrm{R}=7 \cdot 10^{4}$ at $\mathrm{m} / \mathrm{z} 200$ ). Ionization of the LC eluent was carried out using electrospray ionization (ESI) in the negative mode with 40 psi sheath gas $\left(\mathrm{N}_{2}\right)$ and 20 psi aux gas $\left(\mathrm{N}_{2}\right)$. The capillary temperature was set to $350{ }^{\circ} \mathrm{C}$ and a potential of $-3.0 \mathrm{kV}$ was applied to the ESI needle. During each LC run the mass spectrometer operated in full scan mode with a scan range of $m / z 80-550$. Before running the measurements, the instrument was calibrated using the Pierce ${ }^{\mathrm{TM}}$ calibration solution (Thermo Scientific, USA). For an accurate calibration of the lower mass range, butyric acid was added to the solution.

The obtained LC-MS data were recorded by XCalibur 2.2 (Thermo Scientific, USA) and further analyzed by Sieve 2.2 (Thermo Scientific, USA) which allowed a non-target screening of the obtained data set. The threshold for signal abundance was set to $2 \cdot 5 \cdot 10^{6}$ a.u. (i.e signal to noise ratio $\geq 3$ ) for the detection of significant signals in the obtained chromatograms after background subtraction by the software. For the elemental formula assignments, the following isotopes and conditions were used: ${ }^{12} \mathrm{C}$ $(0-50),{ }^{1} \mathrm{H}(0-100),{ }^{16} \mathrm{O}(0-40),{ }^{14} \mathrm{~N}(0-4)$ and ${ }^{32} \mathrm{~S}(0-4)$. The mass tolerance was set to $\pm 5 \mathrm{ppm}$. Afterwards, the obtained compound list was checked for chemically unreasonable formula assignments, such as the absence of hydrogen in carbon-containing compounds or impossible $\mathrm{O} / \mathrm{C}$ ratios $(\mathrm{O} / \mathrm{C}<3$; $0.1<\mathrm{H} / \mathrm{C}<6)$. 


\section{S-1.2 Detected signals and assigned compounds}

Table S-1: List of CHO-containing compounds that were identified by UHPLC-(-)ESI-HRMS analysis of the filter samples.

\begin{tabular}{|c|c|c|c|c|c|c|}
\hline $\begin{array}{l}\text { formula for } \\
\qquad[\mathrm{M}-\mathrm{H}]^{-}\end{array}$ & $\begin{array}{l}m / z \text { for } \\
{[\mathrm{M}-\mathrm{H}]^{-}}\end{array}$ & $\begin{array}{c}\text { measured } \\
\mathrm{m} / \mathrm{z}\end{array}$ & $\begin{array}{l}\Delta m / \\
\text { ppm }\end{array}$ & $\begin{array}{c}\text { Number of } \\
\text { Oxygen }\end{array}$ & $\begin{array}{c}\text { Number of } \\
\text { Carbon }\end{array}$ & O:C \\
\hline $\mathrm{C}_{4} \mathrm{H}_{5} \mathrm{O}_{5}$ & 133.0142 & 133.0141 & -1.1 & 5 & 4 & 1.3 \\
\hline $\mathrm{C}_{6} \mathrm{H}_{7} \mathrm{O}_{4}$ & 143.0350 & 143.0349 & -0.6 & 4 & 6 & 0.7 \\
\hline $\mathrm{C}_{6} \mathrm{H}_{9} \mathrm{O}_{4}$ & 145.0506 & 145.0506 & -0.2 & 4 & 6 & 0.7 \\
\hline $\mathrm{C}_{6} \mathrm{H}_{9} \mathrm{O}_{5}$ & 161.0455 & 161.0454 & -0.9 & 5 & 6 & 0.8 \\
\hline $\mathrm{C}_{6} \mathrm{H}_{13} \mathrm{O}_{6}$ & 181.0718 & 181.0718 & 0.2 & 6 & 6 & 1.0 \\
\hline $\mathrm{C}_{6} \mathrm{H}_{7} \mathrm{O}_{7}$ & 191.0197 & 191.0197 & -0.1 & 7 & 6 & 1.2 \\
\hline $\mathrm{C}_{7} \mathrm{H}_{9} \mathrm{O}_{3}$ & 141.0557 & 141.0557 & -0.1 & 3 & 7 & 0.4 \\
\hline $\mathrm{C}_{7} \mathrm{H}_{9} \mathrm{O}_{4}$ & 157.0506 & 157.0505 & -0.9 & 4 & 7 & 0.6 \\
\hline $\mathrm{C}_{7} \mathrm{H}_{11} \mathrm{O}_{4}$ & 159.0663 & 159.0661 & -1.2 & 4 & 7 & 0.6 \\
\hline $\mathrm{C}_{7} \mathrm{H}_{9} \mathrm{O}_{5}$ & 173.0455 & 173.0453 & -1.4 & 5 & 7 & 0.7 \\
\hline $\mathrm{C}_{7} \mathrm{H}_{11} \mathrm{O}_{5}$ & 175.0612 & 175.0611 & -0.6 & 5 & 7 & 0.7 \\
\hline $\mathrm{C}_{7} \mathrm{H}_{7} \mathrm{O}_{6}$ & 187.0248 & 187.0247 & -0.6 & 6 & 7 & 0.9 \\
\hline $\mathrm{C}_{7} \mathrm{H}_{9} \mathrm{O}_{6}$ & 189.0405 & 189.0403 & -0.9 & 6 & 7 & 0.9 \\
\hline $\mathrm{C}_{8} \mathrm{H}_{13} \mathrm{O}_{3}$ & 157.0870 & 157.0871 & 0.5 & 3 & 8 & 0.4 \\
\hline $\mathrm{C}_{8} \mathrm{H}_{11} \mathrm{O}_{4}$ & 171.0663 & 171.0662 & -0.5 & 4 & 8 & 0.5 \\
\hline $\mathrm{C}_{8} \mathrm{H}_{11} \mathrm{O}_{5}$ & 187.0612 & 187.061 & -1.1 & 5 & 8 & 0.6 \\
\hline $\mathrm{C}_{8} \mathrm{H}_{13} \mathrm{O}_{5} *$ & 189.0768 & 189.0767 & -0.8 & 5 & 8 & 0.6 \\
\hline $\mathrm{C}_{8} \mathrm{H}_{9} \mathrm{O}_{6}$ & 201.0405 & 201.0403 & -0.8 & 6 & 8 & 0.8 \\
\hline $\mathrm{C}_{8} \mathrm{H}_{11} \mathrm{O}_{6} *$ & 203.0561 & 203.0561 & -0.1 & 6 & 8 & 0.8 \\
\hline $\mathrm{C}_{9} \mathrm{H}_{7} \mathrm{O}_{4}$ & 179.0350 & 179.0351 & 0.7 & 4 & 9 & 0.4 \\
\hline $\mathrm{C}_{9} \mathrm{H}_{13} \mathrm{O}_{4}$ & 185.0819 & 185.0818 & -0.7 & 4 & 9 & 0.4 \\
\hline $\mathrm{C}_{9} \mathrm{H}_{11} \mathrm{O}_{5}$ & 199.0612 & 199.0612 & 0.0 & 5 & 9 & 0.6 \\
\hline $\mathrm{C}_{9} \mathrm{H}_{13} \mathrm{O}_{5}$ & 201.0768 & 201.0768 & -0.2 & 5 & 9 & 0.6 \\
\hline $\mathrm{C}_{9} \mathrm{H}_{15} \mathrm{O}_{5}$ & 203.0925 & 203.0925 & 0.0 & 5 & 9 & 0.6 \\
\hline $\mathrm{C}_{9} \mathrm{H}_{9} \mathrm{O}_{6}$ & 213.0405 & 213.0405 & 0.2 & 6 & 9 & 0.7 \\
\hline $\mathrm{C}_{9} \mathrm{H}_{11} \mathrm{O}_{6}$ & 215.0561 & 215.0555 & -2.9 & 6 & 9 & 0.7 \\
\hline $\mathrm{C}_{9} \mathrm{H}_{13} \mathrm{O}_{6}$ & 217.0718 & 217.0716 & -0.8 & 6 & 9 & 0.7 \\
\hline $\mathrm{C}_{9} \mathrm{H}_{9} \mathrm{O}_{7}$ & 229.0354 & 229.0348 & -2.5 & 7 & 9 & 0.8 \\
\hline $\mathrm{C}_{9} \mathrm{H}_{11} \mathrm{O}_{7}$ & 231.0510 & 231.0505 & -2.3 & 7 & 9 & 0.8 \\
\hline $\mathrm{C}_{10} \mathrm{H}_{15} \mathrm{O}_{3}$ & 183.1027 & 183.1026 & -0.4 & 3 & 10 & 0.3 \\
\hline $\mathrm{C}_{10} \mathrm{H}_{13} \mathrm{O}_{5}$ & 213.0768 & 213.0768 & -0.2 & 5 & 10 & 0.5 \\
\hline $\mathrm{C}_{10} \mathrm{H}_{15} \mathrm{O}_{5}$ & 215.0925 & 215.0924 & -0.5 & 5 & 10 & 0.5 \\
\hline $\mathrm{C}_{10} \mathrm{H}_{13} \mathrm{O}_{6}$ & 229.0718 & 229.0715 & -1.2 & 6 & 10 & 0.6 \\
\hline $\mathrm{C}_{10} \mathrm{H}_{15} \mathrm{O}_{6}$ & 231.0874 & 231.0872 & -0.9 & 6 & 10 & 0.6 \\
\hline $\mathrm{C}_{10} \mathrm{H}_{11} \mathrm{O}_{7}$ & 243.0510 & 243.0506 & -1.8 & 7 & 10 & 0.7 \\
\hline
\end{tabular}




\begin{tabular}{ccccccc}
$\mathrm{C}_{10} \mathrm{H}_{15} \mathrm{O}_{7}$ & 247.0823 & 247.0822 & -0.5 & 7 & 10 & 0.7 \\
$\mathrm{C}_{11} \mathrm{H}_{15} \mathrm{O}_{6}$ & 243.0874 & 243.0873 & -0.5 & 6 & 11 & 0.5 \\
$\mathrm{C}_{11} \mathrm{H}_{17} \mathrm{O}_{6}$ & 245.1031 & 245.103 & -0.3 & 6 & 11 & 0.5 \\
$\mathrm{C}_{12} \mathrm{H}_{19} \mathrm{O}_{5}$ & 243.1238 & 243.1237 & -0.4 & 5 & 12 & 0.4 \\
$\mathrm{C}_{12} \mathrm{H}_{21} \mathrm{O}_{5}$ & 245.1394 & 245.1392 & -1.0 & 5 & 12 & 0.4 \\
$\mathrm{C}_{13} \mathrm{H}_{19} \mathrm{O}_{5}$ & 255.1238 & 255.1239 & 0.4 & 5 & 13 & 0.4 \\
$\mathrm{C}_{13} \mathrm{H}_{19} \mathrm{O}_{6}$ & 271.1187 & 271.1188 & 0.3 & 6 & 13 & 0.5 \\
$\mathrm{C}_{14} \mathrm{H}_{21} \mathrm{O}_{5} *$ & 269.1394 & 269.1394 & -0.2 & 5 & 14 & 0.4 \\
$\mathrm{C}_{17} \mathrm{H}_{25} \mathrm{O}_{8}$ & 357.1555 & 357.1559 & 1.1 & 8 & 17 & 0.5 \\
\hline
\end{tabular}

*isobaric compounds detected

Table S-2: List of CHONS-containing compounds that were identified by UHPLC-(-)ESI-HRMS analysis of the filter samples.

\begin{tabular}{ccccccc}
\hline $\begin{array}{c}\text { formula for } \\
{\left[\mathbf{M}-\mathbf{H}^{-}\right.}\end{array}$ & $\begin{array}{c}\boldsymbol{m} / \boldsymbol{z} \text { for } \\
{[\mathbf{M}-\mathbf{H}]^{-}}\end{array}$ & $\begin{array}{c}\text { measured } \\
\boldsymbol{m} / \boldsymbol{z}\end{array}$ & $\begin{array}{c}\boldsymbol{\Delta} \boldsymbol{m} / \\
\mathbf{p p m}\end{array}$ & $\begin{array}{c}\text { Number of } \\
\text { Oxygen }\end{array}$ & $\begin{array}{c}\text { Number of } \\
\text { Carbon }\end{array}$ & O:C \\
\hline $\mathrm{C}_{5} \mathrm{H}_{10} \mathrm{O}_{9} \mathrm{NS}$ & 260.0082 & 260.0079 & -1.1 & 9 & 5 & 1.8 \\
$\mathrm{C}_{5} \mathrm{H}_{9} \mathrm{O}_{11} \mathrm{~N}_{2} \mathrm{~S}$ & 304.9933 & 304.9932 & -0.2 & 11 & 5 & 2.2 \\
$\mathrm{C}_{6} \mathrm{H}_{10} \mathrm{O}_{9} \mathrm{NS}$ & 272.0082 & 272.0081 & -0.3 & 9 & 6 & 1.5 \\
$\mathrm{C}_{7} \mathrm{H}_{10} \mathrm{O}_{9} \mathrm{NS}$ & 284.0082 & 284.0080 & -0.6 & 9 & 7 & 1.3 \\
$\mathrm{C}_{7} \mathrm{H}_{10} \mathrm{O}_{10} \mathrm{NS}$ & 300.0031 & 300.0029 & -0.6 & 10 & 7 & 1.4 \\
$\mathrm{C}_{10} \mathrm{H}_{16} \mathrm{O}_{7} \mathrm{NS}$ & 294.0653 & 294.0657 & 1.4 & 7 & 10 & 0.7 \\
$\mathrm{C}_{10} \mathrm{H}_{14} \mathrm{O}_{8} \mathrm{NS}$ & 308.0446 & 308.0448 & 0.8 & 8 & 10 & 0.8 \\
$\mathrm{C}_{10} \mathrm{H}_{16} \mathrm{O}_{8} \mathrm{NS}$ & 310.0602 & 310.0605 & 0.9 & 8 & 10 & 0.8 \\
$\mathrm{C}_{10} \mathrm{H}_{16} \mathrm{O}_{9} \mathrm{NS}$ & 326.0551 & 326.0551 & -0.1 & 9 & 10 & 0.9 \\
$\mathrm{C}_{10} \mathrm{H}_{16} \mathrm{O}_{10} \mathrm{NS}$ & 342.0500 & 342.0497 & -1.0 & 10 & 10 & 1.0 \\
$\mathrm{C}_{10} \mathrm{H}_{18} \mathrm{O}_{10} \mathrm{NS}$ & 344.0657 & 344.0655 & -0.6 & 10 & 10 & 1.0 \\
$\mathrm{C}_{10} \mathrm{H}_{17} \mathrm{O}_{11} \mathrm{~N} 2 \mathrm{~S}$ & 373.0559 & 373.0558 & -0.2 & 11 & 10 & 1.1 \\
$\mathrm{C}_{10} \mathrm{H}_{15} \mathrm{O}_{12} \mathrm{~N} \mathrm{~S}$ & 387.0351 & 387.0350 & -0.3 & 12 & 10 & 1.2 \\
\hline
\end{tabular}

Table S-3: List of CHON-containing compounds that were identified by UHPLC-(-)ESI-HRMS analysis of the filter samples.

\begin{tabular}{ccccccc}
\hline $\begin{array}{c}\text { formula for } \\
{\left[\mathbf{M}-\mathbf{H}^{-}\right.}\end{array}$ & $\begin{array}{c}\boldsymbol{m} / \boldsymbol{z} \text { for } \\
{\left[\mathbf{M}-\mathbf{H}^{-}\right.}\end{array}$ & $\begin{array}{c}\boldsymbol{m e a s u r e d} \\
\boldsymbol{m} / \boldsymbol{z}\end{array}$ & $\begin{array}{c}\boldsymbol{\Delta} \boldsymbol{m} / \\
\mathbf{p p m}\end{array}$ & $\begin{array}{c}\text { Number of } \\
\text { Oxygen }\end{array}$ & $\begin{array}{c}\text { Number of } \\
\text { Carbon }\end{array}$ & O:C \\
\hline $\mathrm{C}_{7} \mathrm{H}_{4} \mathrm{O}_{5} \mathrm{~N}$ & 182.0095 & 182.0096 & 0.6 & 5 & 7 & 0.7 \\
$\mathrm{C}_{7} \mathrm{H}_{3} \mathrm{O}_{7} \mathrm{~N}_{2}$ & 226.9946 & 226.9947 & 0.5 & 7 & 7 & 1.0 \\
$\mathrm{C}_{10} \mathrm{H}_{16} \mathrm{O}_{8} \mathrm{~N}$ & 278.0881 & 278.0882 & 0.2 & 8 & 10 & 0.8 \\
$\mathrm{C}_{11} \mathrm{H}_{18} \mathrm{O}_{9} \mathrm{~N}$ & 308.0987 & 308.0988 & 0.3 & 9 & 11 & 0.8 \\
\hline
\end{tabular}


Table S-4: List of CHOS-containing compounds that were identified by UHPLC-(-)ESI-HRMS analysis of the filter samples.

\begin{tabular}{|c|c|c|c|c|c|c|}
\hline $\begin{array}{c}\text { formula for } \\
{[\mathrm{M}-\mathrm{H}]^{-}}\end{array}$ & $\begin{array}{c}m / z \text { for } \\
{[\mathrm{M}-\mathrm{H}]^{-}}\end{array}$ & $\begin{array}{c}\text { measured } \\
m / z\end{array}$ & $\Delta m / \mathbf{p p m}$ & $\begin{array}{c}\text { Number of } \\
\text { Oxygen }\end{array}$ & $\begin{array}{c}\text { Number of } \\
\text { Carbon }\end{array}$ & O:C \\
\hline $\mathrm{C}_{2} \mathrm{H}_{3} \mathrm{O}_{6} \mathrm{~S}$ & 154.9656 & 154.9656 & 0.1 & 6 & 2 & 3.0 \\
\hline $\mathrm{C}_{3} \mathrm{H}_{5} \mathrm{O}_{6} \mathrm{~S}$ & 168.9812 & 168.9812 & -0.2 & 6 & 3 & 2.0 \\
\hline $\mathrm{C}_{4} \mathrm{H}_{7} \mathrm{O}_{6} \mathrm{~S}$ & 182.9969 & 182.9967 & -1.0 & 6 & 4 & 1.5 \\
\hline $\mathrm{C}_{5} \mathrm{H}_{9} \mathrm{O}_{6} \mathrm{~S}$ & 197.0125 & 197.0124 & -0.7 & 6 & 5 & 1.2 \\
\hline $\mathrm{C}_{5} \mathrm{H}_{11} \mathrm{O}_{6} \mathrm{~S}$ & 199.0282 & 199.0280 & -0.9 & 6 & 5 & 1.2 \\
\hline $\mathrm{C}_{5} \mathrm{H}_{7} \mathrm{O}_{7} \mathrm{~S}$ & 210.9918 & 210.9916 & -0.9 & 7 & 5 & 1.4 \\
\hline $\mathrm{C}_{5} \mathrm{H}_{9} \mathrm{O}_{7} \mathrm{~S}$ & 213.0074 & 213.0075 & 0.2 & 7 & 5 & 1.4 \\
\hline $\mathrm{C}_{5} \mathrm{H}_{11} \mathrm{O}_{7} \mathrm{~S}$ & 215.0231 & 215.0229 & -0.9 & 7 & 5 & 1.4 \\
\hline $\mathrm{C}_{5} \mathrm{H}_{7} \mathrm{O}_{8} \mathrm{~S}$ & 226.9867 & 226.9865 & -0.9 & 8 & 5 & 1.6 \\
\hline $\mathrm{C}_{6} \mathrm{H}_{11} \mathrm{O}_{6} \mathrm{~S}$ & 211.0282 & 211.0280 & -0.9 & 6 & 6 & 1.0 \\
\hline $\mathrm{C}_{7} \mathrm{H}_{11} \mathrm{O}_{6} \mathrm{~S}$ & 223.0282 & 223.0280 & -0.8 & 6 & 7 & 0.9 \\
\hline $\mathrm{C}_{7} \mathrm{H}_{11} \mathrm{O}_{7} \mathrm{~S}$ & 239.0231 & 239.0231 & 0.0 & 7 & 7 & 1.0 \\
\hline $\mathrm{C}_{7} \mathrm{H}_{13} \mathrm{O}_{7} \mathrm{~S}$ & 241.0387 & 241.0385 & -1.0 & 7 & 7 & 1.0 \\
\hline $\mathrm{C}_{7} \mathrm{H}_{7} \mathrm{O}_{8} \mathrm{~S}$ & 250.9867 & 250.9868 & 0.3 & 8 & 7 & 1.1 \\
\hline $\mathrm{C}_{7} \mathrm{H}_{9} \mathrm{O}_{8} \mathrm{~S}$ & 253.0024 & 253.0028 & 1.7 & 8 & 7 & 1.1 \\
\hline $\mathrm{C}_{8} \mathrm{H}_{13} \mathrm{O}_{7} \mathrm{~S}$ & 253.0387 & 253.0384 & -1.4 & 7 & 8 & 0.9 \\
\hline $\mathrm{C}_{8} \mathrm{H}_{11} \mathrm{O}_{9} \mathrm{~S}$ & 283.0129 & 283.0127 & -0.8 & 9 & 8 & 1.1 \\
\hline $\mathrm{C}_{8} \mathrm{H}_{13} \mathrm{O}_{9} \mathrm{~S}$ & 285.0286 & 285.0284 & -0.6 & 9 & 8 & 1.1 \\
\hline $\mathrm{C}_{8} \mathrm{H}_{13} \mathrm{O}_{10} \mathrm{~S}$ & 301.0235 & 301.0231 & -1.3 & 10 & 8 & 1.3 \\
\hline $\mathrm{C}_{9} \mathrm{H}_{15} \mathrm{O}_{6} \mathrm{~S}$ & 251.0595 & 251.0593 & -0.7 & 6 & 9 & 0.7 \\
\hline $\mathrm{C}_{9} \mathrm{H}_{15} \mathrm{O}_{7} \mathrm{~S}$ & 267.0543 & 267.0543 & 0.0 & 7 & 9 & 0.8 \\
\hline $\mathrm{C}_{9} \mathrm{H}_{13} \mathrm{O}_{8} \mathrm{~S}$ & 281.0337 & 281.0334 & -0.9 & 8 & 9 & 0.9 \\
\hline $\mathrm{C}_{9} \mathrm{H}_{13} \mathrm{O}_{9} \mathrm{~S}$ & 297.0286 & 297.0282 & -1.3 & 9 & 9 & 1.0 \\
\hline $\mathrm{C}_{10} \mathrm{H}_{17} \mathrm{O}_{5} \mathrm{~S}$ & 249.0802 & 249.0801 & -0.5 & 5 & 10 & 0.5 \\
\hline $\mathrm{C}_{10} \mathrm{H}_{15} \mathrm{O}_{7} \mathrm{~S}$ & 279.0544 & 279.0544 & 0.0 & 7 & 10 & 0.7 \\
\hline $\mathrm{C}_{10} \mathrm{H}_{17} \mathrm{O}_{7} \mathrm{~S}$ & 281.0700 & 281.0698 & -0.9 & 7 & 10 & 0.7 \\
\hline $\mathrm{C}_{10} \mathrm{H}_{17} \mathrm{O}_{8} \mathrm{~S}$ & 297.0650 & 297.0646 & -1.2 & 8 & 10 & 0.8 \\
\hline $\mathrm{C}_{10} \mathrm{H}_{15} \mathrm{O}_{9} \mathrm{~S}$ & 311.0442 & 311.0440 & -0.7 & 9 & 10 & 0.9 \\
\hline $\mathrm{C}_{10} \mathrm{H}_{17} \mathrm{O}_{9} \mathrm{~S}$ & 313.0599 & 313.0596 & -0.9 & 9 & 10 & 0.9 \\
\hline $\mathrm{C}_{10} \mathrm{H}_{15} \mathrm{O}_{10} \mathrm{~S}$ & 327.0391 & 327.0387 & -1.4 & 10 & 10 & 1.0 \\
\hline $\mathrm{C}_{10} \mathrm{H}_{13} \mathrm{O}_{11} \mathrm{~S}$ & 341.0184 & 341.0183 & -0.3 & 11 & 10 & 1.1 \\
\hline $\mathrm{C}_{11} \mathrm{H}_{19} \mathrm{O}_{7} \mathrm{~S}$ & 295.0857 & 295.0858 & 0.3 & 7 & 11 & 0.6 \\
\hline
\end{tabular}




\section{S-2. Trajectory calculations for the campaign period}
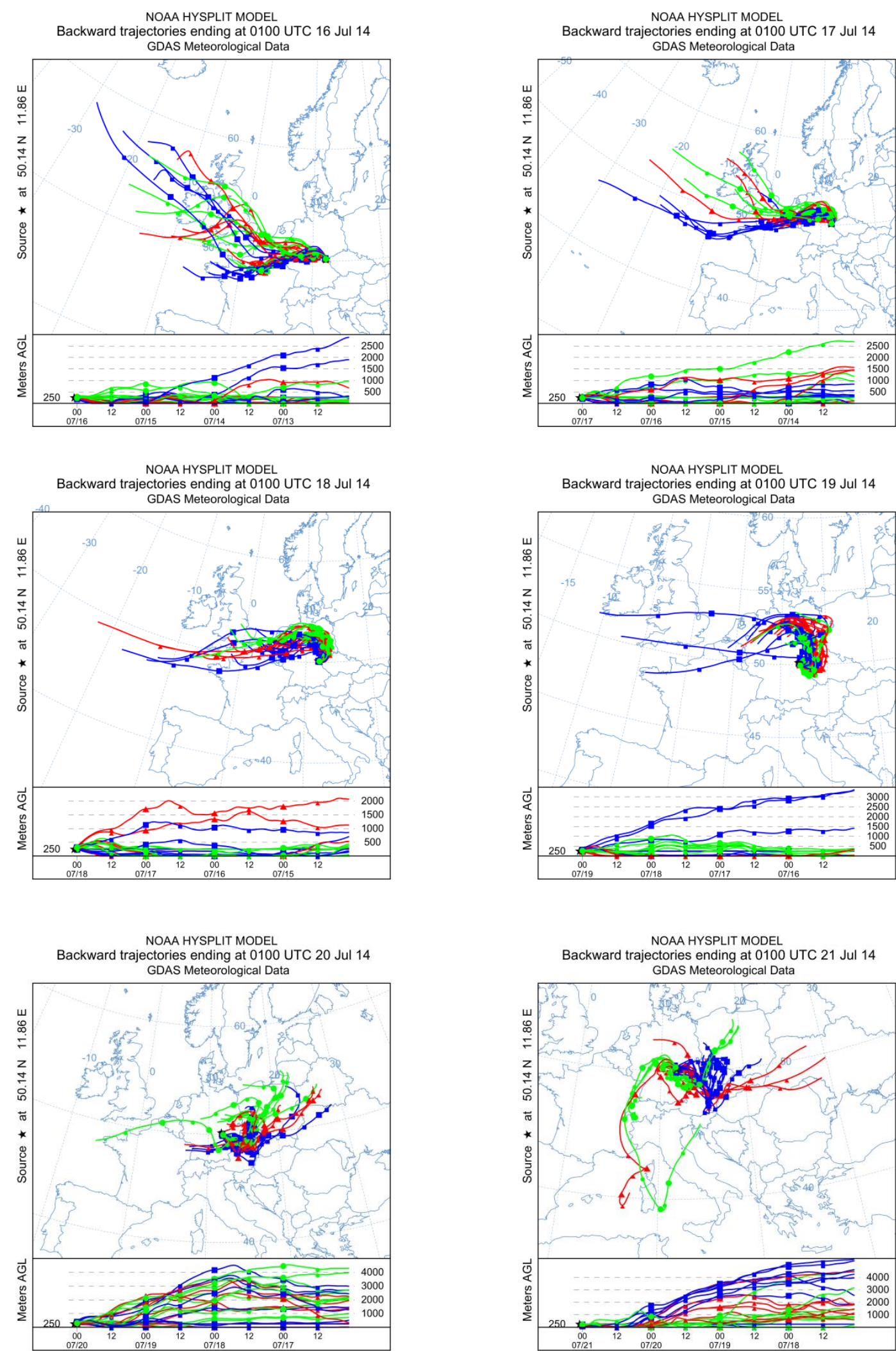

Figure S-1: 96 hours backward HYSPLIT trajectory calculations for the $16^{\text {th }}-21^{\text {st }}$ of July (each at 12 midnight CET) (Draxler and Rolph). 
NOAA HYSPLIT MODEL

Backward trajectories ending at 0100 UTC 22 Jul 14 GDAS Meteorological Data

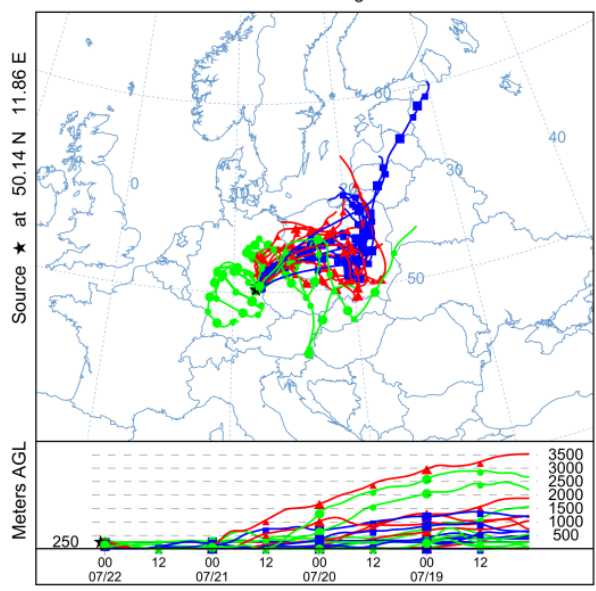

NOAA HYSPLIT MODE

UTC 24 Jul 14 GDAS Meteorological Data

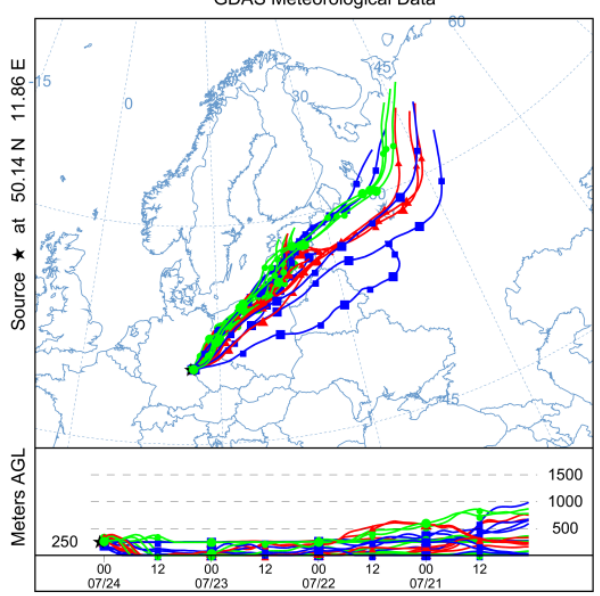

Backward trajec

ctories ending at 0100 UTC 26 Jul 14 AS Meteorological Dat

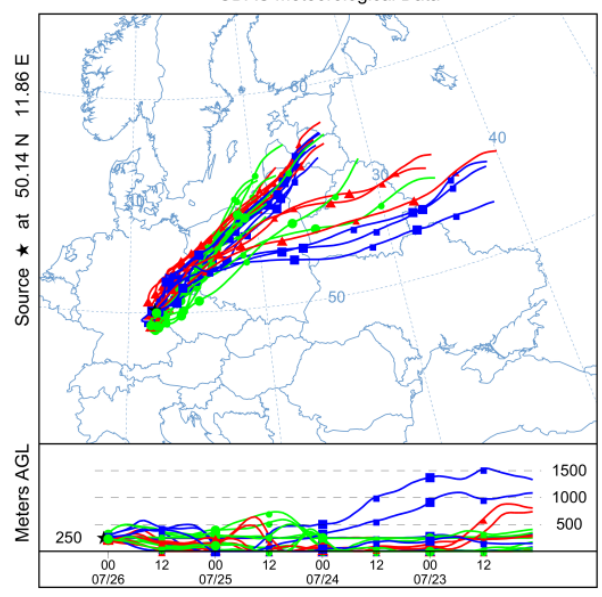

NOAA HYSPLIT MODEL

Backward trajectoris ending at 0100 UTC 23 Jul 14 GDAS Meteorological Data

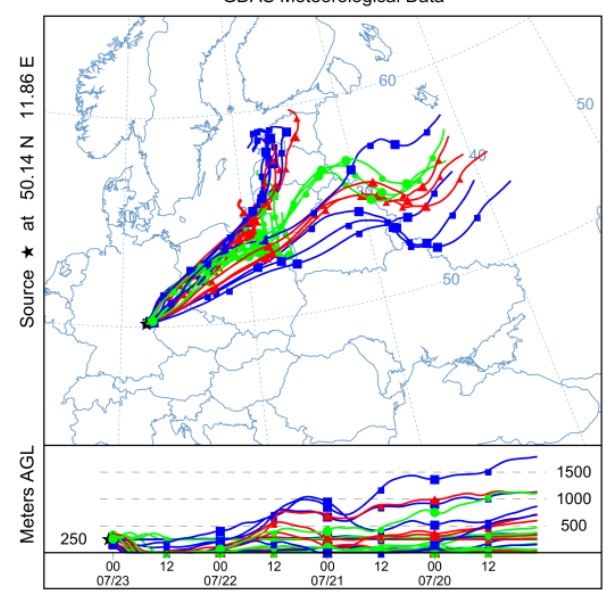

NOAA HYSPLIT MODEL

00 UTC 25 Jul 14 GDAS Meteorological Data

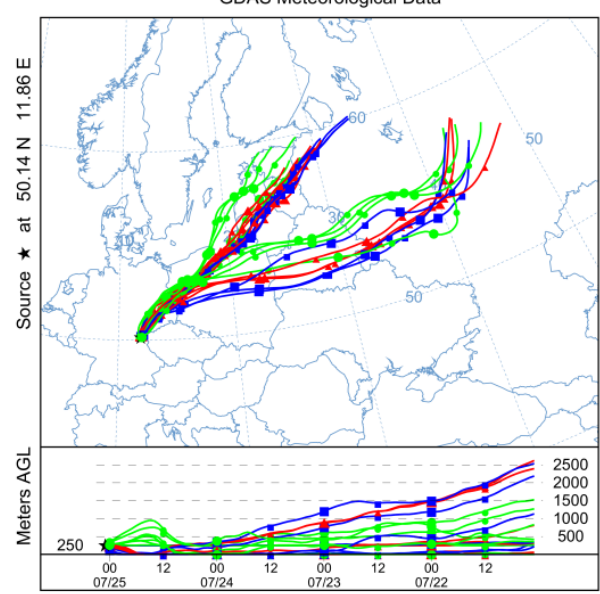

NOAA HYSPLIT MODEL

Backward trajectories ending at 0100 UTC 27 Jul 14 GDAS Meteorological Dat

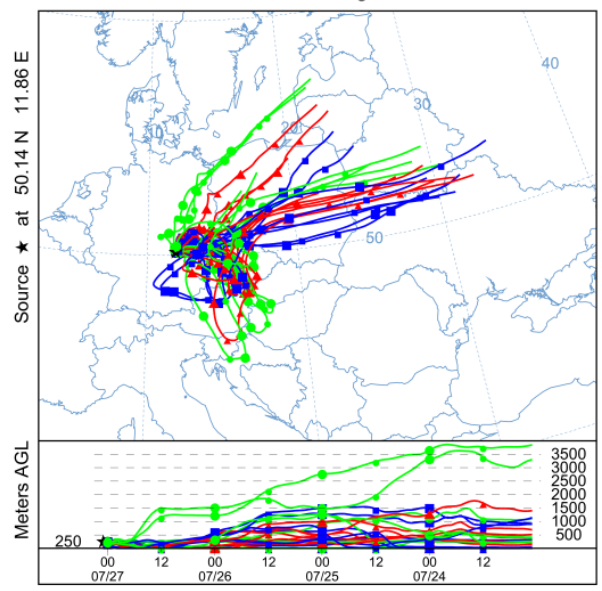

Figure S-2: 96 hours backward HYSPLIT trajectory calculations for the $22^{\text {nd }}-27^{\text {th }}$ of July (each at 12 midnight CET) (Draxler and Rolph). 


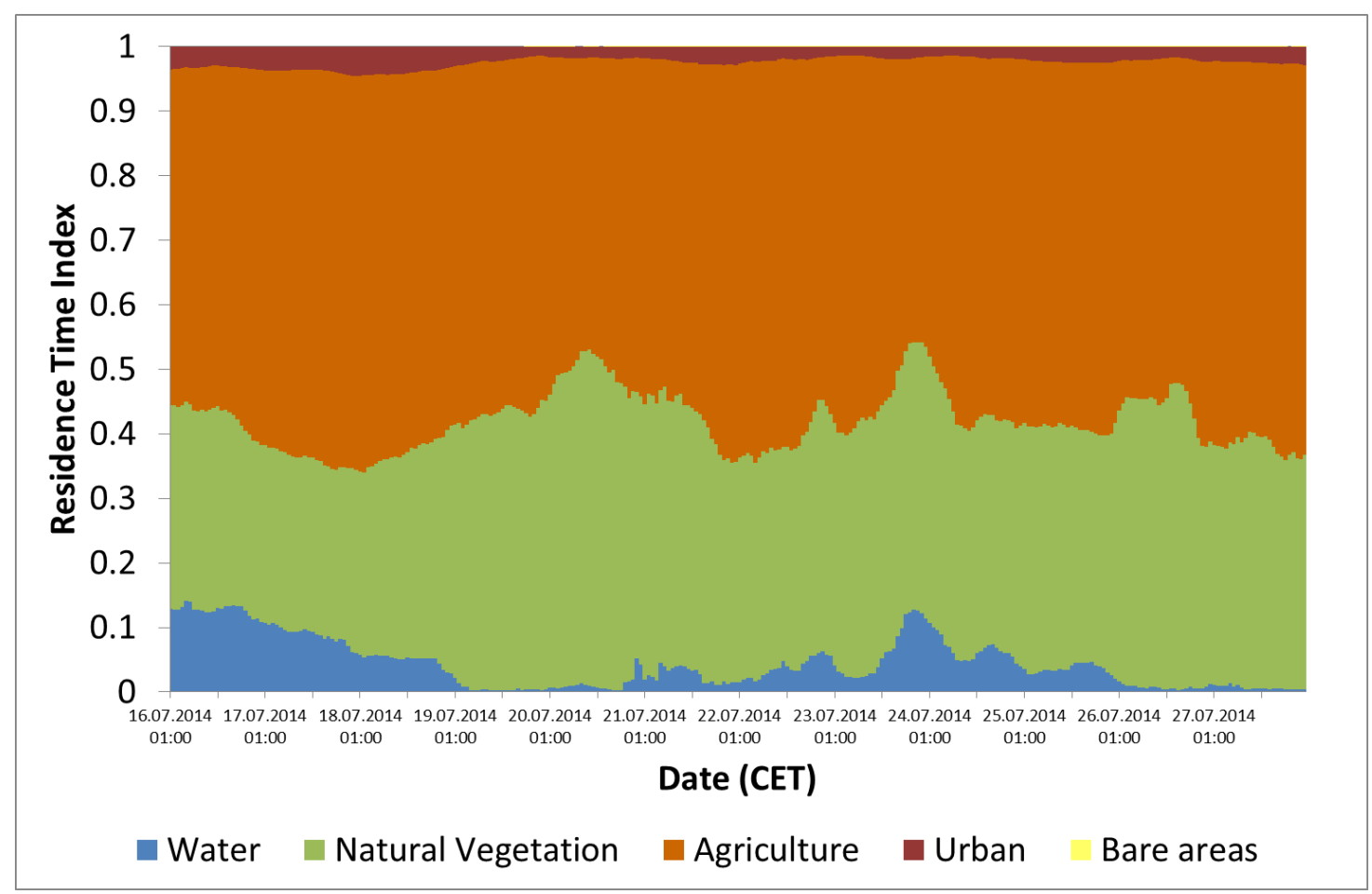

Figure S-3: Residence times for 96 hours backward trajectories arriving at the site intersected with satellite-derived global landcover data to give indications of influences of main land cover classes. A detailed description of the calculation method can be found elsewhere (van Pinxteren et al., 2010).

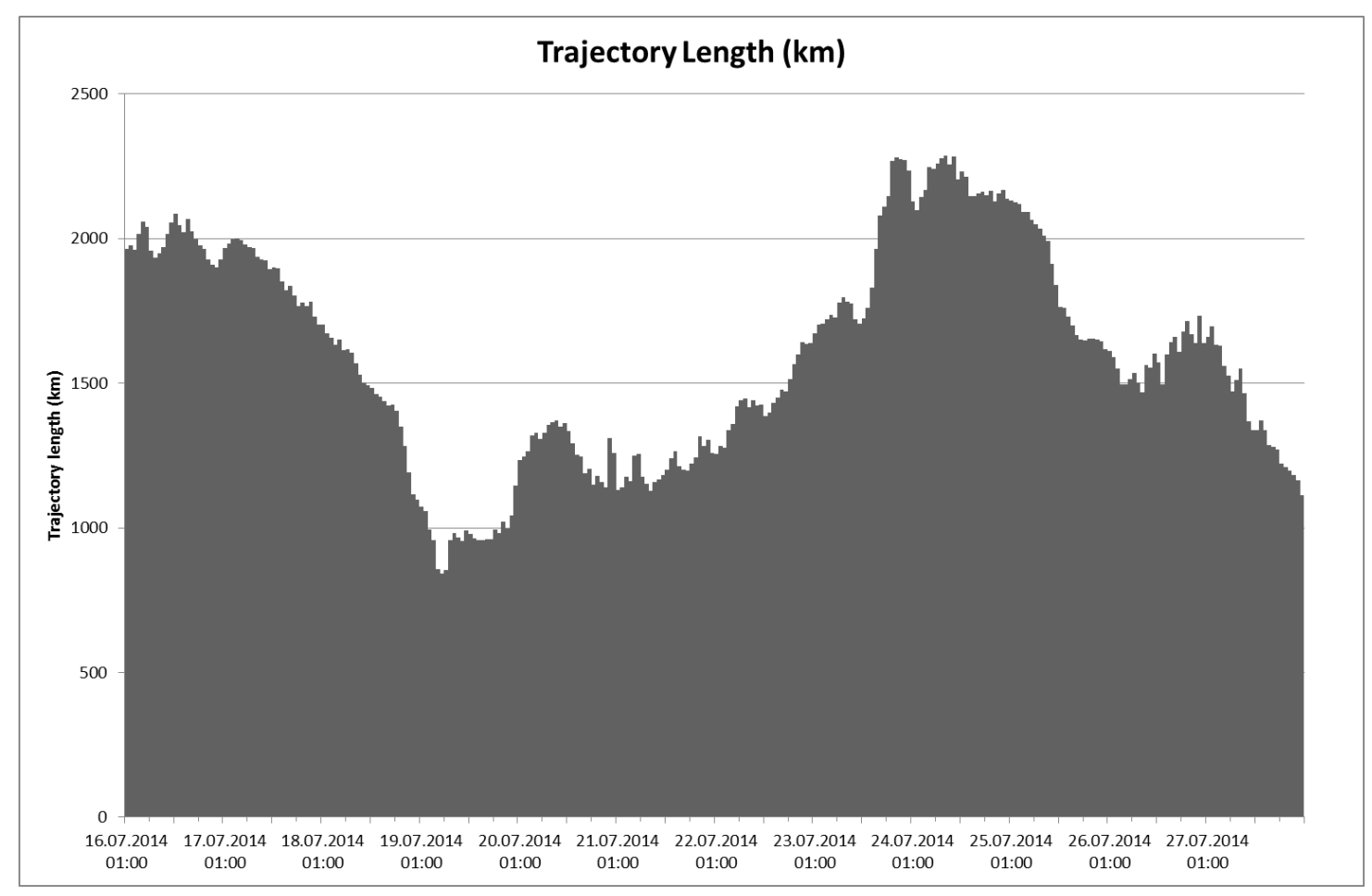

Figure S-4: Trajectory lengths for 96 hours backward trajectories arriving at the site. For details see van Pinxteren et al. (2010). 


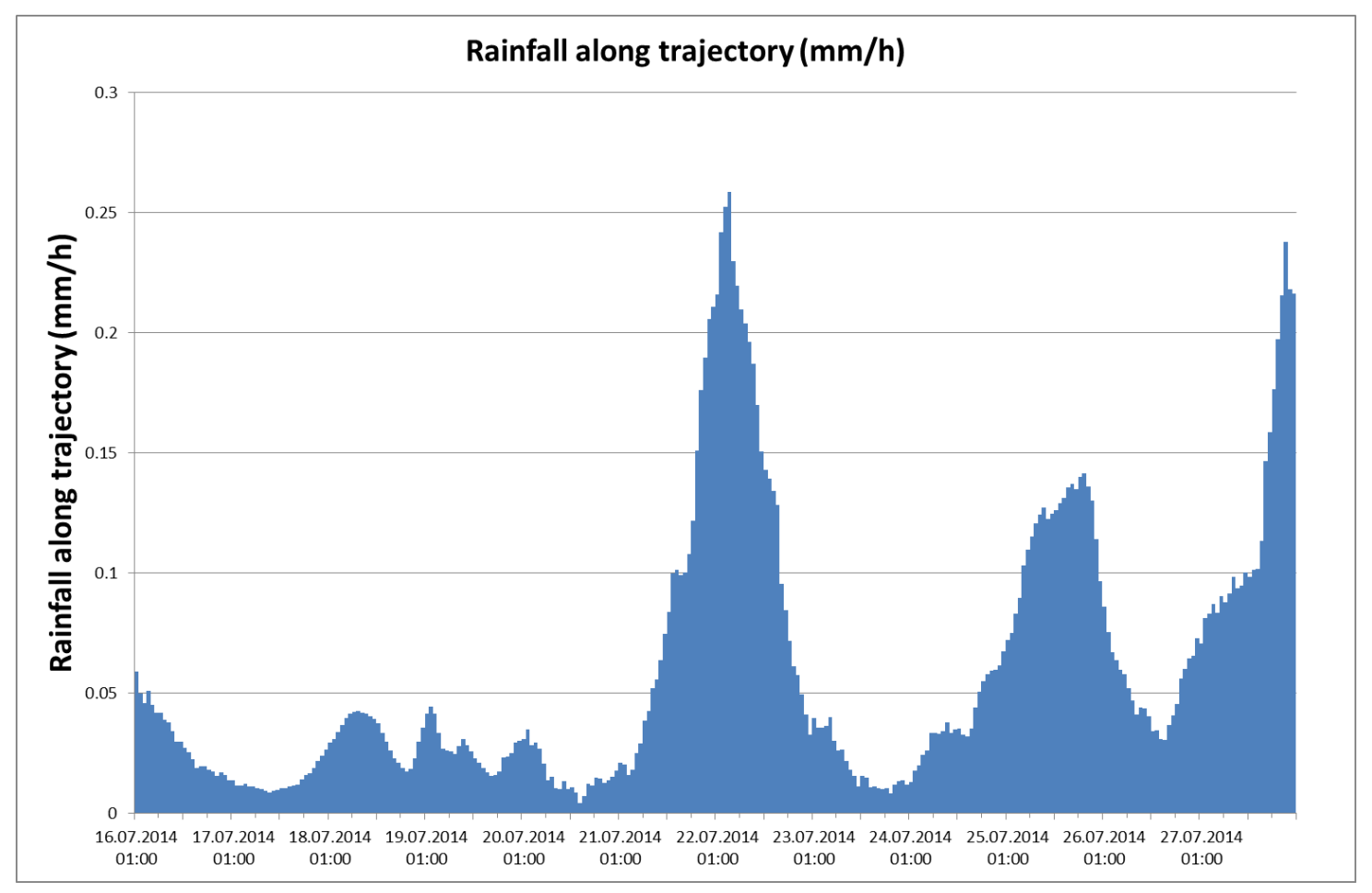

Figure S-5: Rainfall along the calculated 96 hours backward trajectories arriving at the site. For details see van Pinxteren et al. (2010).

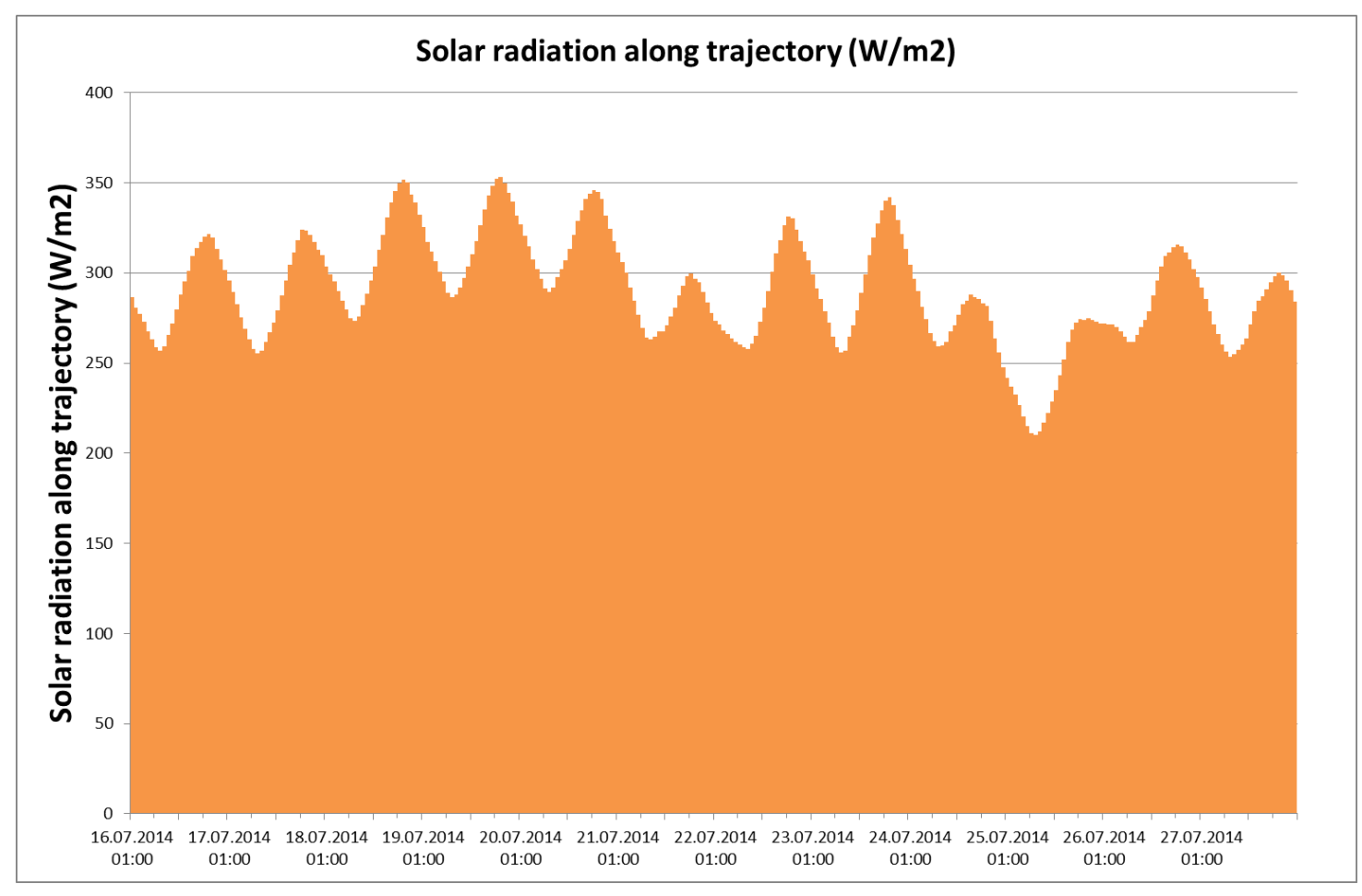

Figure S-6: Solar radiation along the calculated 96 hours backward trajectories arriving at the site. For details see van Pinxteren et al. (2010). 


\section{S-3. Supplementary mass spectrometric data}

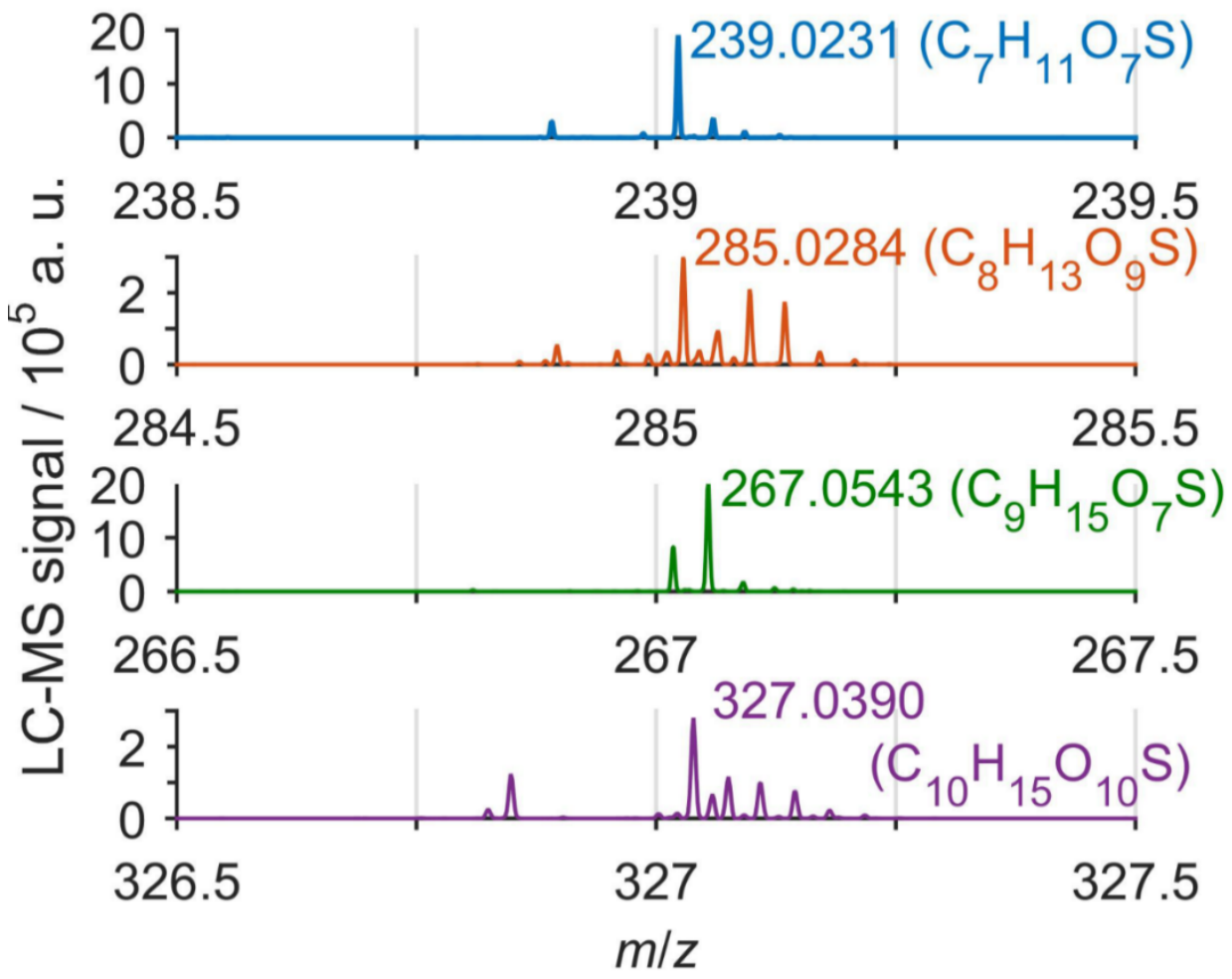

Figure S-7: Mass spectra of the LC-MS analysis of a filter sample (mass resolution of $\mathrm{R}=7 \cdot 10^{4}$ at $\mathrm{m} / \mathrm{z} 200$ ). Four HOOS compounds were chosen as representative according to their number of carbon atoms $\left(\mathrm{C}_{7}-\mathrm{C}_{10}\right)$ and signal abundance.
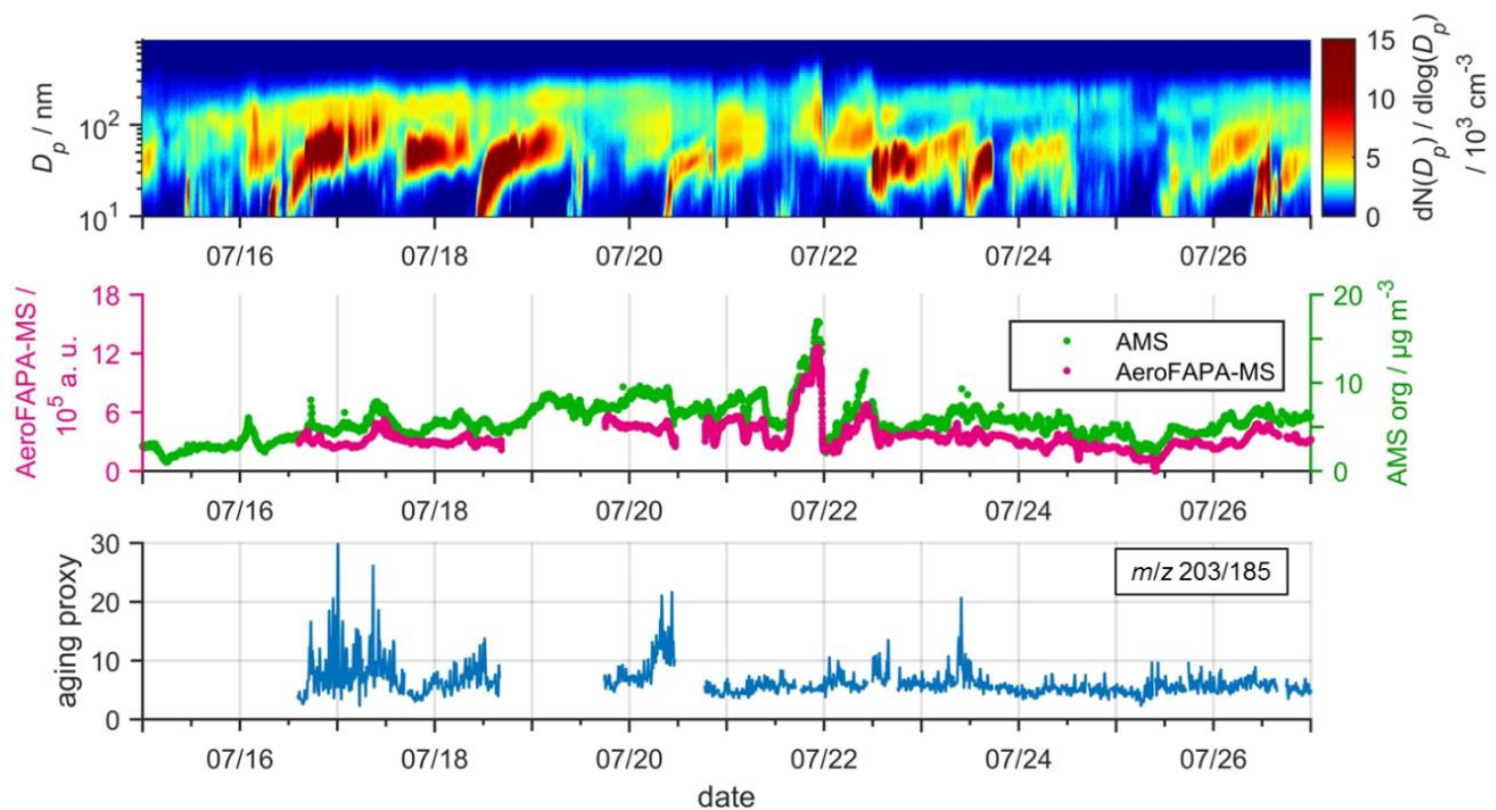

Figure S-8: Top panel: Number size distribution of aerosol particles which was measured by an SMPS. Middle panel: Time traces of the total ion current of the AeroFAPA-MS (magenta) and the organic aerosol mass measured by an AMS (green). Bottom panel: Ratio of $m / z$ 203/185 as aging proxy for SOA particles at the site, measured by the AeroFAPA-MS. 


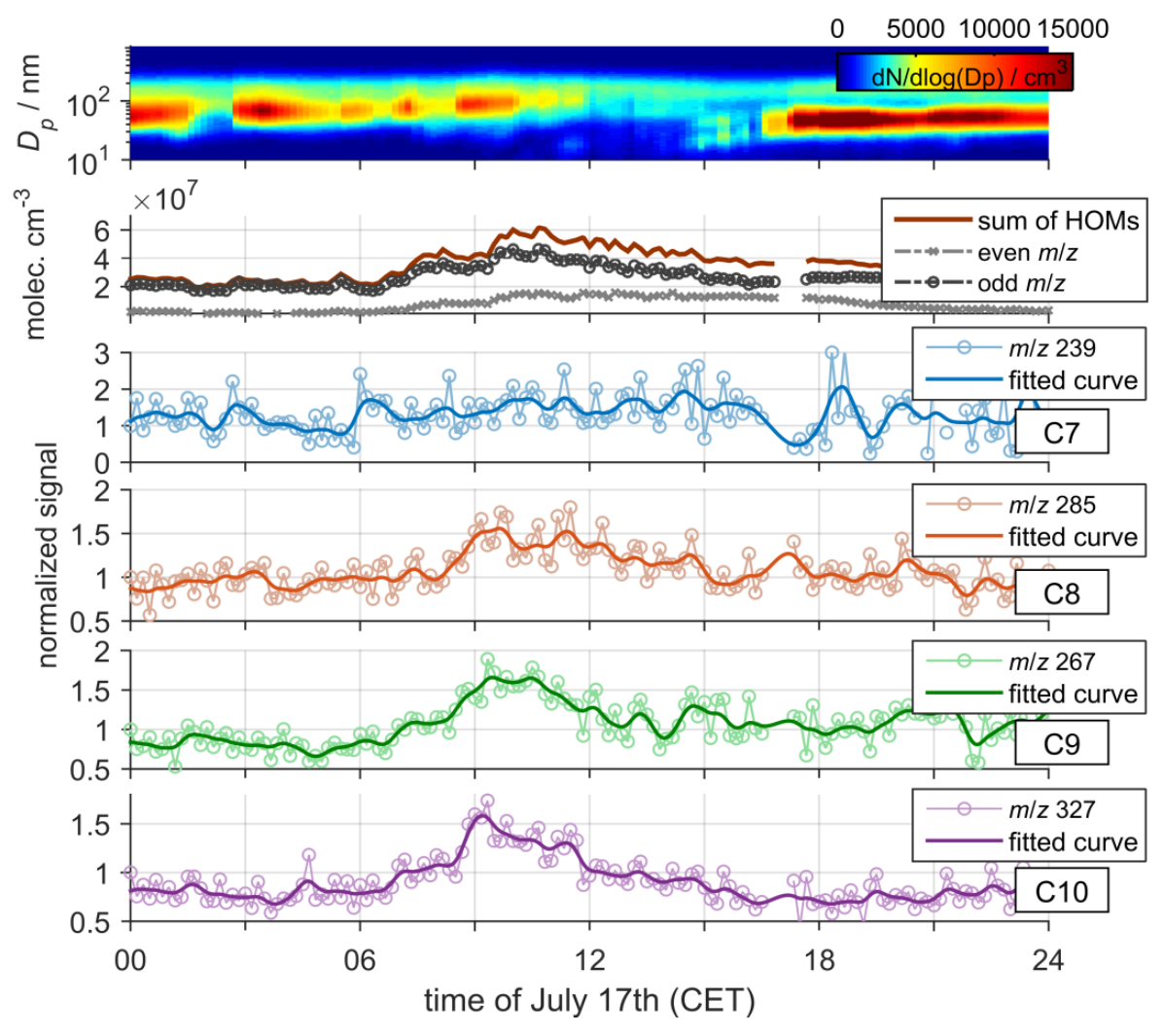

Figure S-9: Time traces for C7-C10 HOOS, gas-phase HOMs and particle number size distribution during July $17^{\text {th }}$. HOM concentration is dominated by ions with odd $m / z$ ratios $\left(\left[\mathrm{M}+\mathrm{NO}_{3}\right]^{-}\right)$, indicating the presence of peroxyradicals $\left(\mathrm{RO}_{2}{ }^{\circ}\right)$, organonitrates $\left(\mathrm{RONO}_{2}\right)$ and peroxynitrates $\left(\mathrm{RO}_{2} \mathrm{NO}_{2}\right)$. While the larger HOOS (i.e. C7, $\mathrm{C} 8, \mathrm{C} 10$ ) are following the trend of the HOM signals with odd $\mathrm{m} / \mathrm{z}$ ratios, the C7 HOOS differ from this behavior, further supporting the assumption that these species are not directly formed. In contrast to the online data, filter samples show the highest abundance for the class of C7 HOOS. Thus, C7 HOOS might represent decomposition products of the larger HOOS.

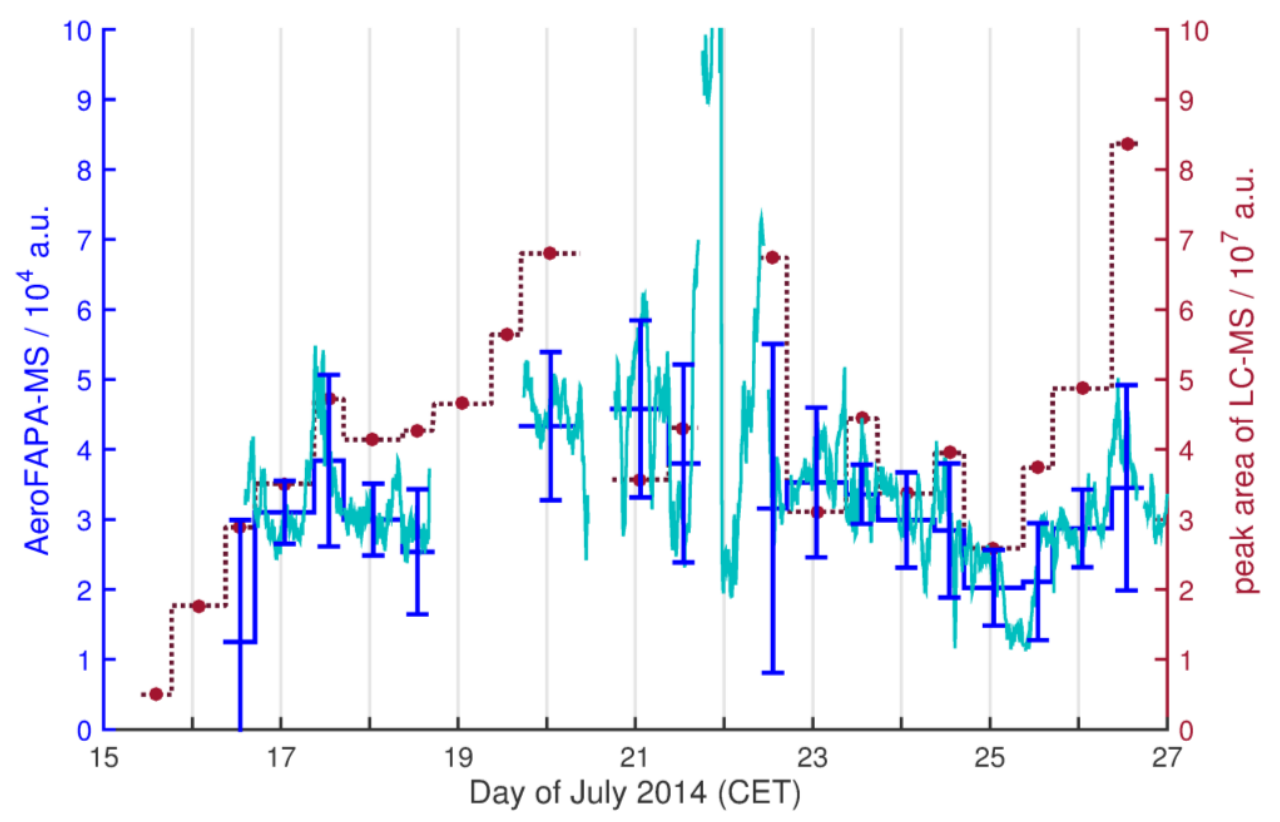

Figure S-10: Comparison of the signals for the sum of HOOS detected by AeroFAPA-MS (light blue), and by LC-MS (red). The signals of the AeroFAPA-MS are averaged for the filter sampling times (dark blue); error bars depict one standard deviation. 

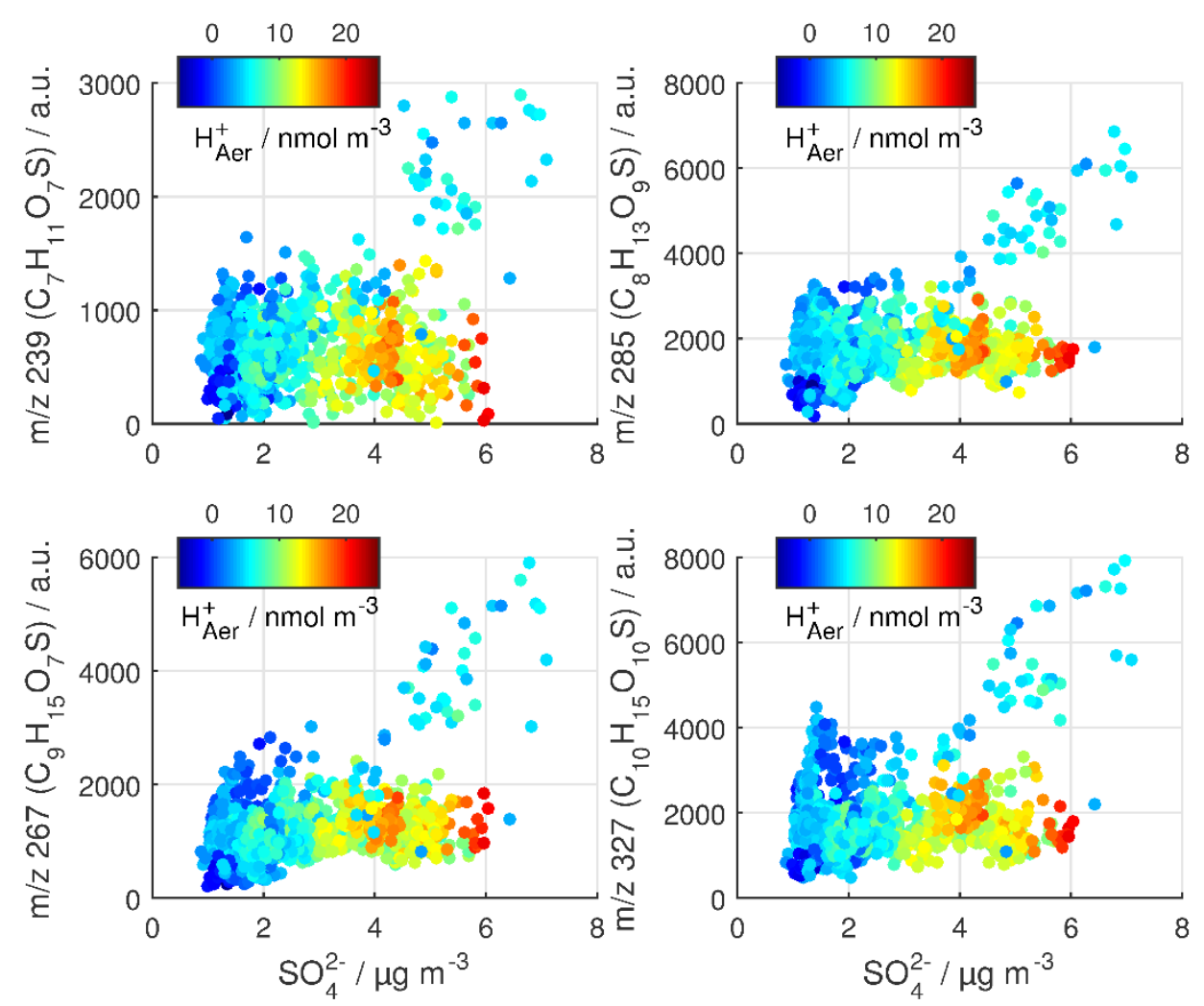

Figure S-11: Correlations between HOOS signals (AeroFAPA-MS data), particulate sulfate concentrations (AMS data), and aerosol acidity $\left(\mathrm{H}_{\text {eer }}{ }^{+}\right.$, AMS data). For the entire campaign period the particle acidity was very low and rather stable (average of $\mathrm{H}^{+}$Aer $=7.4 \mathrm{nmol} \mathrm{m}^{-3}$ ), indicating the presence of partially or even fully neutralized particles. Aerosol acidity was estimated by calculating the concentrations of $\mathrm{H}_{\text {eer }}{ }^{+}$from AMS data according to Zhang et al. (2007).

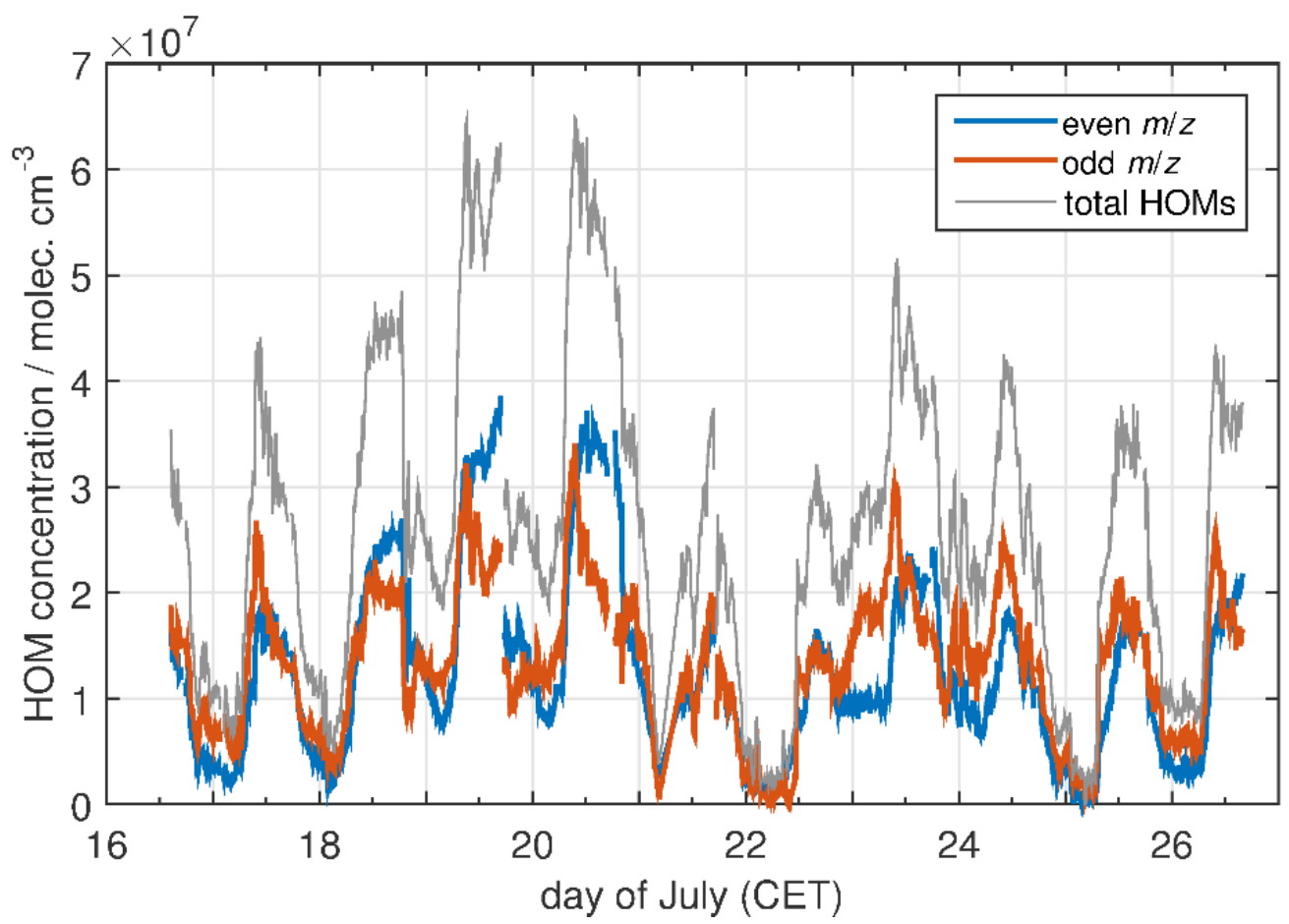

Figure S-12: Concentrations of gas-phase HOMs measured by the CI-APi-TOFMS over the entire campaign period. 
Table S-5: Signals and assignments for gas-phase HOMs detected by CI-APi-TOFMS.

\begin{tabular}{|c|c|c|c|c|}
\hline formula assignment & classification & $\begin{array}{c}\text { molecular } \\
\text { weight }\end{array}$ & $\begin{array}{c}m / z \text { for } \\
{\left[\mathrm{M}+\mathrm{NO}_{3}\right]^{-}}\end{array}$ & reference \\
\hline $\mathrm{C}_{7} \mathrm{H}_{10} \mathrm{O}_{4}$ & HOM & 158 & 220 & Ehn et al., 2014 \\
\hline $\mathrm{C}_{10} \mathrm{H}_{15} \mathrm{O}_{6}$ & $\mathrm{RO}_{2}$ radical & 231 & 293 & Jokinen et al., 2014 \\
\hline- & - & 232 & 294 & \\
\hline- & - & 233 & 295 & \\
\hline- & - & 235 & 297 & \\
\hline $\mathrm{C}_{8} \mathrm{H}_{12} \mathrm{O}_{8}$ & HOM & 236 & 298 & Ehn et al., 2014 \\
\hline- & - & 245 & 307 & \\
\hline $\mathrm{C}_{10} \mathrm{H}_{14} \mathrm{O}_{7}$ & HOM & 246 & 308 & Ehn et al., 2014 \\
\hline- & - & 247 & 309 & \\
\hline $\mathrm{C}_{9} \mathrm{H}_{12} \mathrm{O}_{8} / \mathrm{C}_{10} \mathrm{H}_{16} \mathrm{O}_{7}$ & HOM & 248 & 310 & Ehn et al., 2014 \\
\hline $\mathrm{C}_{10} \mathrm{H}_{17} \mathrm{O}_{7}$ & $\mathrm{RO}_{2}$ radical & 249 & 311 & Jokinen et al., 2014 \\
\hline $\mathrm{C}_{10} \mathrm{H}_{15} \mathrm{O}_{8}$ & $\mathrm{RO}_{2}$ radical & 263 & 325 & Jokinen et al., 2014 \\
\hline $\mathrm{C}_{10} \mathrm{H}_{16} \mathrm{O}_{8} / \mathrm{C}_{9} \mathrm{H}_{12} \mathrm{O}_{9}$ & HOM & 264 & 326 & Ehn et al., 2014 \\
\hline- & - & 265 & 327 & \\
\hline- & - & 267 & 329 & \\
\hline- & $\begin{array}{c}\mathrm{RO}_{2} \mathrm{NO}_{2} \\
\left(m / z 293+\mathrm{NO}_{2}\right)\end{array}$ & 277 & 339 & Jokinen et al., 2014 \\
\hline $\mathrm{C}_{10} \mathrm{H}_{14} \mathrm{O}_{9}$ & HOM & 278 & 340 & Ehn et al., 2014 \\
\hline $\mathrm{C}_{10} \mathrm{H}_{16} \mathrm{O}_{9}$ & HOM & 280 & 342 & Ehn et al., 2014 \\
\hline- & $\begin{array}{c}\mathrm{RONO}_{2} \\
(m / z 325+\mathrm{NO})\end{array}$ & 293 & 355 & Jokinen et al., 2014 \\
\hline $\mathrm{C}_{10} \mathrm{H}_{15} \mathrm{O}_{10}$ & $\mathrm{RO}_{2}$ radical & 295 & 357 & Jokinen et al., 2014 \\
\hline $\mathrm{C}_{10} \mathrm{H}_{16} \mathrm{O}_{10}$ & HOM & 296 & 358 & Ehn et al., 2014 \\
\hline- & - & 308 & 370 & \\
\hline $\mathrm{C}_{10} \mathrm{H}_{14} \mathrm{O}_{11}$ & HOM & 310 & 372 & Ehn et al., 2014 \\
\hline $\mathrm{C}_{10} \mathrm{H}_{16} \mathrm{O}_{11}$ & $\mathrm{HOM}$ & 312 & 374 & Ehn et al., 2014 \\
\hline
\end{tabular}




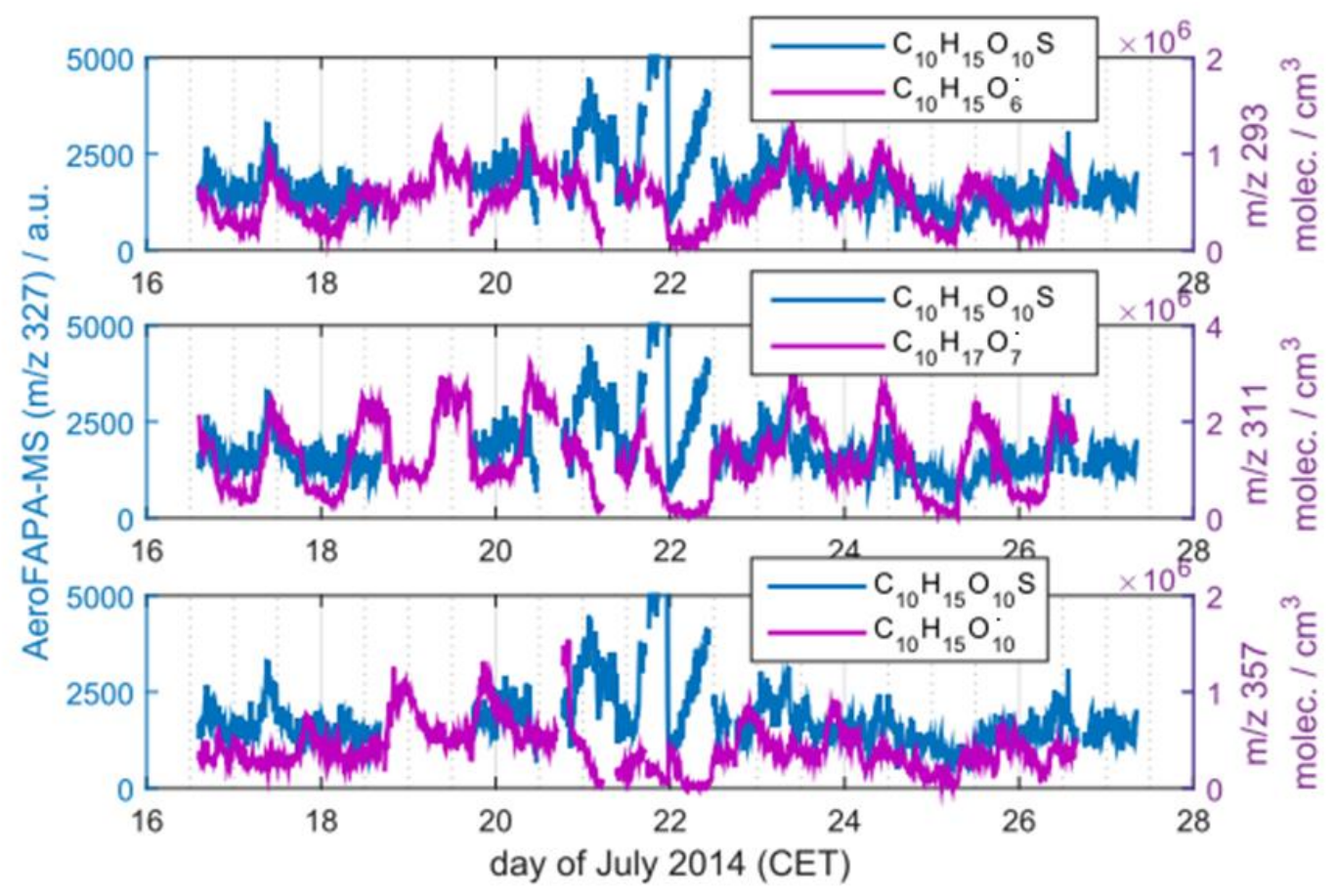

Figure S-13: Time series for the C10 HOOS signals $\left(m / z 327,[\mathrm{M}-\mathrm{H}]^{-}\right)$of the AeroFAPA-MS and $\mathrm{RO}_{2}{ }^{\circ}$ at $m / z 293$, 311 , and $357\left(\left[\mathrm{M}+\mathrm{NO}_{3}\right]^{-}\right)$, measured by the CI-APi-TOFMS. Except for $\mathrm{C}_{10} \mathrm{H}_{15} \mathrm{O}_{10}{ }^{\circ}\left(\mathrm{m} / z\right.$ 357, $\left.\left[\mathrm{M}+\mathrm{NO}_{3}\right]^{-}\right)$, signals for $\mathrm{RO}_{2}{ }^{\circ}$ show similar trends as the HOOS signals. However, the best agreement was observed for the $\mathrm{C}_{10} \mathrm{H}_{15} \mathrm{O}_{8}{ }^{\circ}$ $\left(\mathrm{m} / \mathrm{z} 325,\left[\mathrm{M}+\mathrm{NO}_{3}\right]^{-}\right)$and the signals for $\mathrm{C} 10$ HOOS (see Fig. 6 in the manuscript).

a)
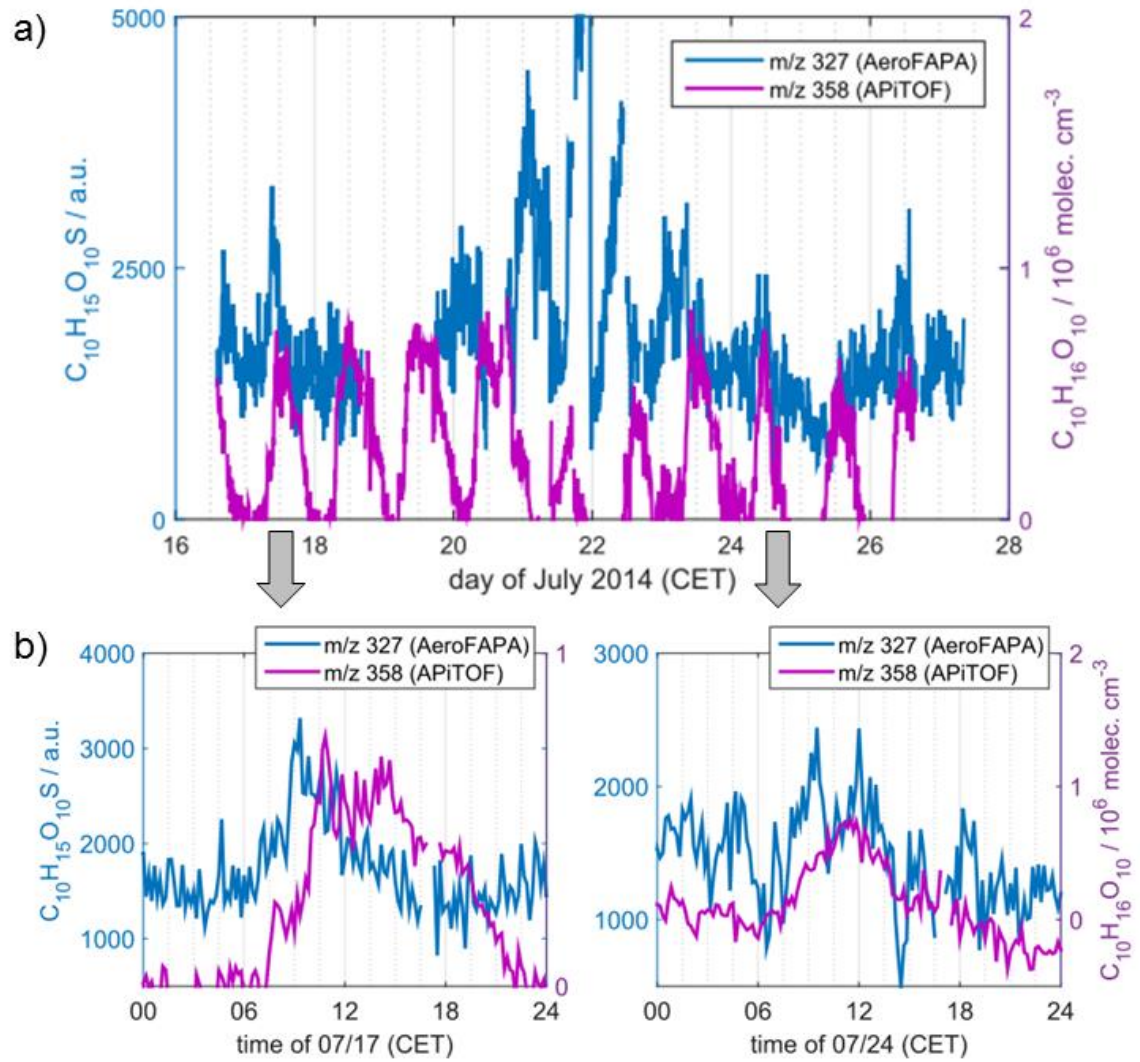

Figure S-14: Time series for the C10 HOOS signals $\left(m / z 327,[\mathrm{M}-\mathrm{H}]^{-}\right)$of the AeroFAPA-MS and CI-APi-TOFMS signal for the closed-shell $\mathrm{HOM} \mathrm{C} \mathrm{C}_{10} \mathrm{H}_{16} \mathrm{O}_{10}\left(\mathrm{~m} / \mathrm{z} 358,\left[\mathrm{M}+\mathrm{NO}_{3}\right]^{-}\right)$. In contrast to the signals for $\mathrm{RO}_{2}{ }^{\circ}$, the signal shows less agreement to the signals for $\mathrm{C} 10$ HOOS (see also Fig. 6 and Fig. S-13). 


\section{References}

Draxler, R. and Rolph, G.: HYSPLIT (HYbrid Single-Particle Lagrangian Integrated Trajectory) Model.

Ehn, M., Thornton, J. A., Kleist, E., Sipila, M., Junninen, H., Pullinen, I., Springer, M., Rubach, F., Tillmann, R., Lee, B., Lopez-Hilfiker, F., Andres, S., Acir, I.-H., Rissanen, M., Jokinen, T., Schobesberger, S., Kangasluoma, J., Kontkanen, J., Nieminen, T., Kurten, T., Nielsen, L. B., Jorgensen, S., Kjaergaard, H. G., Canagaratna, M., Maso, M. D., Berndt, T., Petaja, T., Wahner, A., Kerminen, V.-M., Kulmala, M., Worsnop, D. R., Wildt, J., and Mentel, T. F.: A large source of low-volatility secondary organic aerosol, Nature, 506, 476-479, 2014.

Jokinen, T., Sipilä, M., Richters, S., Kerminen, V.-M., Paasonen, P., Stratmann, F., Worsnop, D., Kulmala, M., Ehn, M., Herrmann, H., and Berndt, T.: Rapid Autoxidation Forms Highly Oxidized RO2 Radicals in the Atmosphere, Angew. Chem. Int. Ed., 53, 14596-14600, doi:10.1002/anie.201408566, 2014.

Riva, M., Da Silva Barbosa, T., Lin, Y.-H., Stone, E. A., Gold, A., and Surratt, J. D.: Chemical characterization of organosulfates in secondary organic aerosol derived from the photooxidation of alkanes, Atmos. Chem. Phys., 16, 11001-11018, doi:10.5194/acp-1611001-2016, 2016.

van Pinxteren, D., Brüggemann, E., Gnauk, T., Müller, K., Thiel, C., and Herrmann, H.: A GIS based approach to back trajectory analysis for the source apportionment of aerosol constituents and its first application, J Atmos Chem, 67, 1-28, doi:10.1007/s10874-0119199-9, 2010.

Zhang, Q., Jimenez, J. L., Worsnop, D. R. and Canagaratna, M.: A Case Study of Urban Particle Acidity and Its Influence on Secondary Organic Aerosol, Environ. Sci. Technol., 41(9), 3213-3219, doi:10.1021/es061812j, 2007. 